\title{
Changing of network characteristics of acrylamide/maleic acid hydrogels by alteration of irradiation dose rate
}

\author{
M. Șen ${ }^{\mathrm{a}, *}$, C. Uzun ${ }^{\mathrm{a}}, \mathrm{A} . \mathrm{Safrany}^{\mathrm{b}}$, O. Kantoḡlu ${ }^{\mathrm{c}}$, O. Güven ${ }^{\mathrm{a}}$ \\ a Polymer Chemistry Division, Department of Chemistry, Hacettepe University, Beytepe, Ankara 06532, Turkey \\ ${ }^{\mathrm{b}}$ Chemical Research Center, Institute of Isotope and Surface Chemistry, Hungarian Academy of Sciences, P. O. B. 77, \\ Budapest H-1525, Hungary \\ ${ }^{\mathrm{c}}$ Food Irradiation and Sterilization Department, Ankara Nuclear Agriculture and Animal Research Center, Turkish Atomic Energy \\ Authority, Ankara 06983, Turkey
}

\begin{abstract}
Poly(acrylamide/maleic acid) P(AAm/MA) hydrogels were prepared by irradiating the ternary mixtures of AAm/MA and water by gamma rays at ambient temperature at very low $(0.18 \mathrm{kGy} / \mathrm{h})$, and moderate dose rate $(3.0 \mathrm{kGy} / \mathrm{h})$. The equilibrium degree of swelling (EDS) of hydrogels prepared at $3.0 \mathrm{kGy} / \mathrm{h}$ dose rate increased from $520 \%$ to $3900 \%$ with increasing MA mole content in the gel system from $0 \%$ to $5.2 \%$. On the other hand, no systematic dependence of swelling on MA content was observed for hydrogels obtained at low dose rate irradiation due to formation of inhomogeneous network structure and large pores in the gel. Pore structure of hydrogels was monitored by using scanning electron microscope. Systematic swelling of P(AAm/MA) hydrogels prepared at moderate dose rates can be explained with homogeneous pore size distribution of network.
\end{abstract}

(C) 2003 Elsevier Science Ltd. All rights reserved.

Keywords: Hydrogel; Poly(acrylamide/maleic acid); Network structure; Dose rate

\section{Introduction}

The most characteristic property of hydrogels is their ability to swell in the presence of water and to shrink in the absence of it. The two most important factors controlling the swelling of hydrogels are the hydrophilicity of polymer chain and the cross-link density. By incorporating stimuli-responsive co-monomers either into the backbone or as pendant group it is possible to prepare hydrogels with responsive properties. Responsive behavior of hydrogels makes them very attractive materials for some specific biomedical applications, or separation processes such as enrichment and purification. Sites showing selectivity for proteins, enzymes, biomolecules or dyes, pigments or metal ions can be easily incorporated into network structure by radiation-

\footnotetext{
*Corresponding author. Tel.: +90-312-2977989; fax: +90312-2977989.

E-mail address: msen@hacettepe.edu.tr (M. Șen).
}

induced polymerization (Sen et al., 2000). We have been using maleic acid and itaconic acid as the co-monomers imparting $\mathrm{pH}$ responsiveness to PVP and acrylamide (AAm) based hydrogels. During the last decade a number of papers published from this laboratory showed clearly the advantages of using radiation processing of aqueous monomer solutions to synthesize co-polymeric PVP and AAm hydrogels with stimuliresponsive properties (Güven et al., 1999). These hydrogels possess the ability to swell, shrink, bend or even degrade in response to $\mathrm{pH}$, temperature, ionic strength and type of salt. The present study aims to make a comparative assessment on the effect of irradiation dose rate on the network structure of AAm/MA hydrogels. Hydrogels with varying maleic acid (MA) content were prepared from the ternary mixtures of AAm/MA/water by irradiation with gamma rays at very low dose rate $(0.18 \mathrm{kGy} / \mathrm{h})$, and moderate dose rate $(3.0 \mathrm{kGy} / \mathrm{h})$. For the investigation of the effect of irradiation dose rate on the hydrogel properties; 
swelling behavior, pore structure, and mechanical behavior were investigated.

\section{Experimental}

The two monomers used in this study, namely, AAm and MA were obtained from Fluka and $\mathrm{BDH}$, respectively. Aqueous solutions of monomers of $1 \mathrm{~g}$ AAm and 20, 40, 60 and $90 \mathrm{mg}$ MA were prepared in $1 \mathrm{ml}$ of pure water in different compositions (AAm/MA mole ratios, 98.8:1.2, 97.6:2.4, 96.5:3.5, 94.8:5.2). PVC straws of $4 \mathrm{~mm}$ diameter were filled with these solutions and placed in a Gammacell 220 type gamma irradiator. The irradiation was done in air at ambient temperature at two dose rates of 0.18 and $3.0 \mathrm{kGy} / \mathrm{h}$ and total dose of $25 \mathrm{kGy}$. The hydrogels obtained in long cylindrical shapes were cut into 3-4 $\mathrm{mm}$ long pieces. The amount of MA in the monomer, polymer and/or copolymer form was determined by titration of extract against $\mathrm{NaOH}(0.05 \mathrm{M})$ to phenolphthalein end point. Dried hydrogels were left to swell in water at $\mathrm{pH} 7.0$ and $25^{\circ} \mathrm{C}$. Swollen gels removed from the water at regular intervals were dried superficially with filter paper, weighed and placed in the same bath. The measurements were continued until a constant weight was reached for each sample. The pore structure was monitored by using Jeol JSM 5600LV model scanning electron microscope. Elastic properties and elastic modulus of hydrogels were determined by using Zwick Z010 model Universal Testing Instrument and uniaxial compression module.

\section{Results and discussion}

\subsection{Preparation of $P(A A m / M A)$ hydrogels}

When pure AAm monomer is irradiated with gamma rays, polymerization and cross-linking reactions take

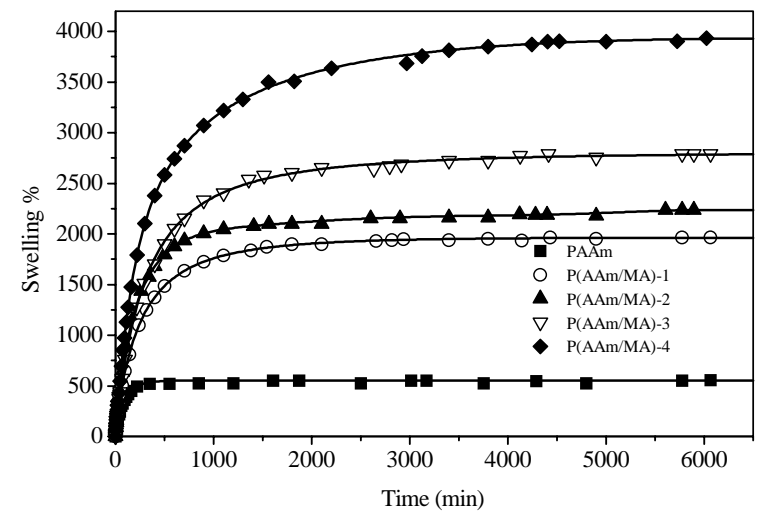

place simultaneously. Total dose required for $100 \%$ gelation of AAm/MA hydrogels has been found to be $7 \mathrm{kGy}$ when MA used in the range of $0.0-5.2 \mathrm{~mol} \%$ in the initial mixture (Akkas, 1996). In this study, ternary mixtures of AAm $/ \mathrm{MA} / \mathrm{H}_{2} \mathrm{O}$ were irradiated to $25 \mathrm{kGy}$ with gamma rays at two dose rates of 0.18 and $3.0 \mathrm{kGy} / \mathrm{h}$. No titrate was consumed for AAm/MA sol fractions, and $100 \%$ gelation was achieved for all AAm/MA mixtures for both irradiation dose rates. Mole percentages of MA in the co-polymeric hydrogels denoted as P(AAm/MA)-1, P(AAm/MA)-2, P(AAm/MA)-3, and $\mathrm{P}(\mathrm{AAm} / \mathrm{MA})-4$ are $1.2,2.4,3.5$ and 5.2, respectively.

\subsection{Swelling behavior of $P(A A m / M A)$ hydrogels}

For the characterization of network structure of prepared hydrogels the swelling properties were first investigated. Fig. 1a and b represents the mass swelling kinetics of AAm/MA hydrogels at $25^{\circ} \mathrm{C}$ in distilled water. Consistently with poly-electrolyte behavior, equilibrium swelling of hydrogels prepared at moderate dose rate was found to increase with MA content in the gel structure. On the other hand, no systematic dependence of swelling on MA content was observed for hydrogels obtained at low dose rate irradiation. This unsystematic behavior was attributed to formation of inhomogeneous network structure and large pores in the gel due to drying of gel surface during long irradiation periods and accumulation of gaseous products inside the gel. The swelling curves of hydrogels were used for the calculation of nature of diffusion of water in the hydrogel by using $F=M_{t} / M_{\infty}=k t^{n}$ equation (Sen et al., 1998). When this equation is applied to the initial stages of swelling, plots of $M_{t} / M_{\infty}$ vs. $\ln t$ yield straight lines. The exponents $n$ and $k$ were calculated from the slope and intercept of the lines and listed in Table 1. The values in this table show that for AAm/MA hydrogels prepared at moderate dose values $n$ assumes values between 0.5 and 0.73 and the higher the amount of MA,

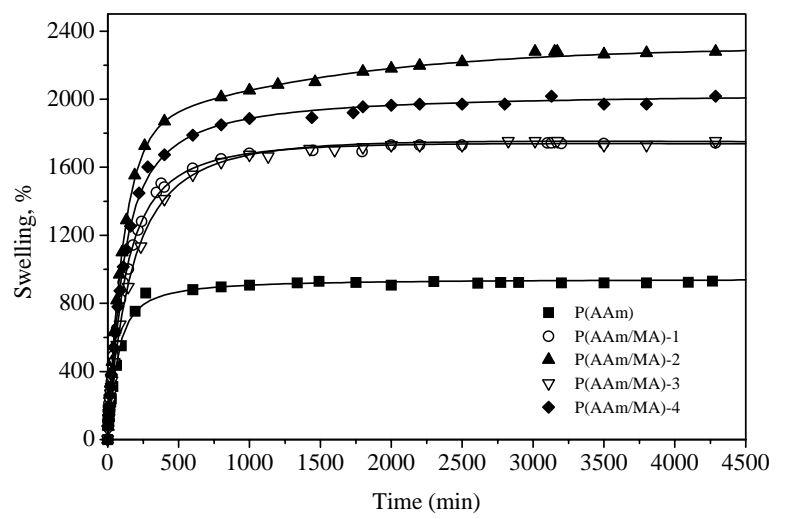

Fig. 1. Swelling kinetics of P(AAm/MA) hydrogels. Irradiation dose rate (a) $3.0 \mathrm{kGy} / \mathrm{h}$, (b) $0.18 \mathrm{kGy} / \mathrm{h}$. 
the more non-Fickian is the transport of water into the hydrogel system. This is generally explained as a consequence of a slow relaxation rate of the polymer matrix. On the other hand, due to inhomogeneous network structure and large pores of hydrogels prepared at low dose rate no systematic dependence of diffusion type on MA content was observed.

\subsection{Pore structure of $P(A A m / M A)$ hydrogels}

Pore structure of hydrogels was monitored by using scanning electron microscope. Representative figures are given in Fig. 2 for P(AAm/MA)-3 hydrogel system. As can be seen from figure pore structure of hydrogels prepared at low dose rate contains very large and irregular pores in the gels (Fig. 2a and b). Very similar pore structure was observed for the other gel compositions prepared at low dose rate. Systematic swelling of $\mathrm{P}(\mathrm{AAm} / \mathrm{MA})$ hydrogels prepared at moderate dose rates can be explained with homogeneous pore size distribution of network (Fig. 2c and d).

\subsection{Mechanical properties of $P(A A m / M A)$ hydrogels}

For the investigation of the effect of dose rate on the mechanical properties of hydrogels uniaxial compres-

Table 1

Swelling characteristics of $\mathrm{P}(\mathrm{AAm} / \mathrm{MA})$ hydrogels

\begin{tabular}{llllll}
\hline Gel name & \multicolumn{2}{l}{ Moderate dose rate } & & \multicolumn{2}{l}{ Low dose rate } \\
\cline { 2 - 3 } & $n$ & $k$ & & $k$ & $k$ \\
\hline PAAm & 0.50 & $5.75 \times 10^{-2}$ & & 0.37 & 0.377 \\
P(AAm/MA)-1 & 0.67 & $1.57 \times 10^{-2}$ & & 0.48 & 0.276 \\
P(AAm/MA)-2 & 0.66 & $1.50 \times 10^{-2}$ & & 0.50 & 0.259 \\
P(AAm/MA)-3 & 0.72 & $9.64 \times 10^{-3}$ & & 0.54 & 0.228 \\
P(AAm/MA)-4 & 0.73 & $9.04 \times 10^{-3}$ & & 0.50 & 0.256 \\
\hline
\end{tabular}

sion was supplied by using the Universal Testing Instrument. Typical strain-stress curves of hydrogels are given in Fig. $3 \mathrm{a}$ and $\mathrm{b}$ for moderate and low dose rates, respectively. As can be seen from the figure while the magnitude of $\%$ strain at deformation increased with increasing MA content, \% stress decreased for hydrogels prepared at moderate dose rate. The results also indicate that there is a close relation between mechanical properties and swelling properties. Fig. 2 and Table 2 show that inhomogeneity of the network structure also effects the mechanical properties of the hydrogel system. Shear moduli values of hydrogels were calculated by using elastic deformation theory and $f=G\left(\lambda-\lambda^{-2}\right)$ equation (Treloar, 1975), where $f$ is the applied force to unit area and $\lambda$ is the deformation ratio equal to $L / L_{0} . L_{0}$ and $L$ are heights of the cylindrical specimens of undeformed and deformed hydrogels during compression, respectively. $G$ is the shear modulus of hydrogel. When equation is applied to the initial stages of deformation, plots of $f$ vs. $\lambda-\lambda^{-2}$ yield straight lines. The $G$ value was calculated from the slope of lines and listed in Table 2.

As shown in Table 2 shear moduli of hydrogels prepared at moderate dose rate continuously decrease due to plasticizing effect of MA in the hydrogel structure. Unsystematic behavior in the shear moduli, strain and stress values at maximum deformation were observed for the low dose rate hydrogel. The shear moduli values retained 5.8-9.2 independent of the amount of MA in the hydrogel. This behavior was attributed again to formation of inhomogeneous network structure and large pores in the gel due to drying of gel surface during long irradiation period and accumulation of gaseous products in the gel structure.

As a conclusion, the swelling, electron microscope and mechanical studies showed that in addition to the relative amount of poly-electrolytic groups and crosslinking density of the system, the irradiation dose rate is

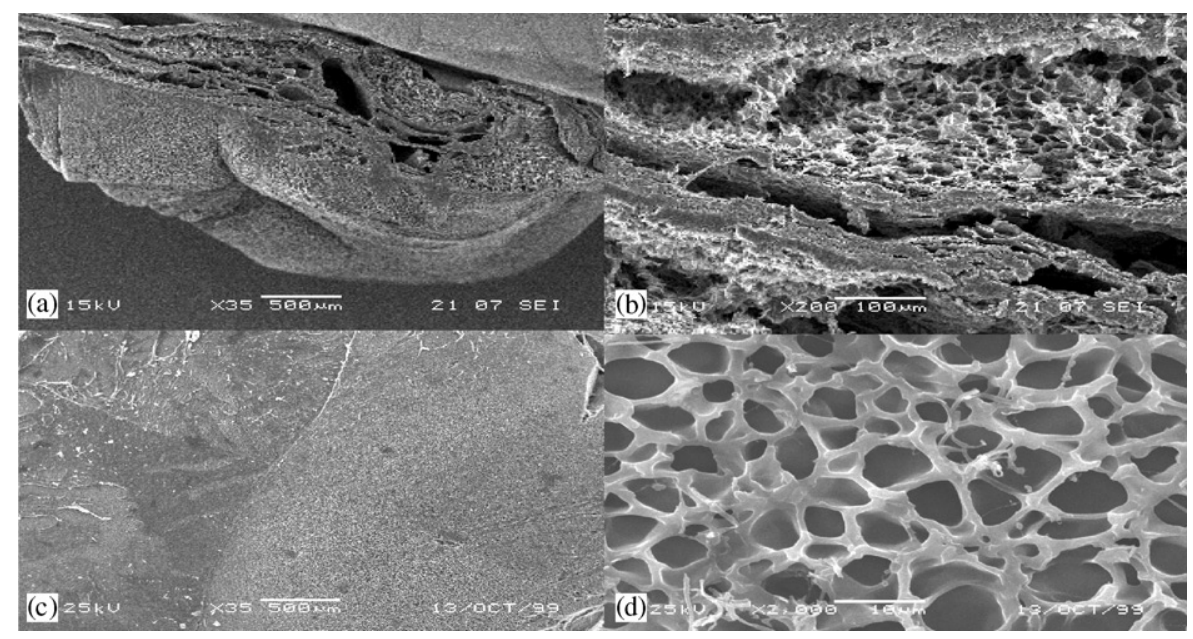

Fig. 2. Pore structure of P(AAm/MA) hydrogels. Irradiation dose rate $0.18 \mathrm{kGy} / \mathrm{h}$ for (a) and (b) and $3.0 \mathrm{kGy} / \mathrm{h}$ for (c) and (d). 

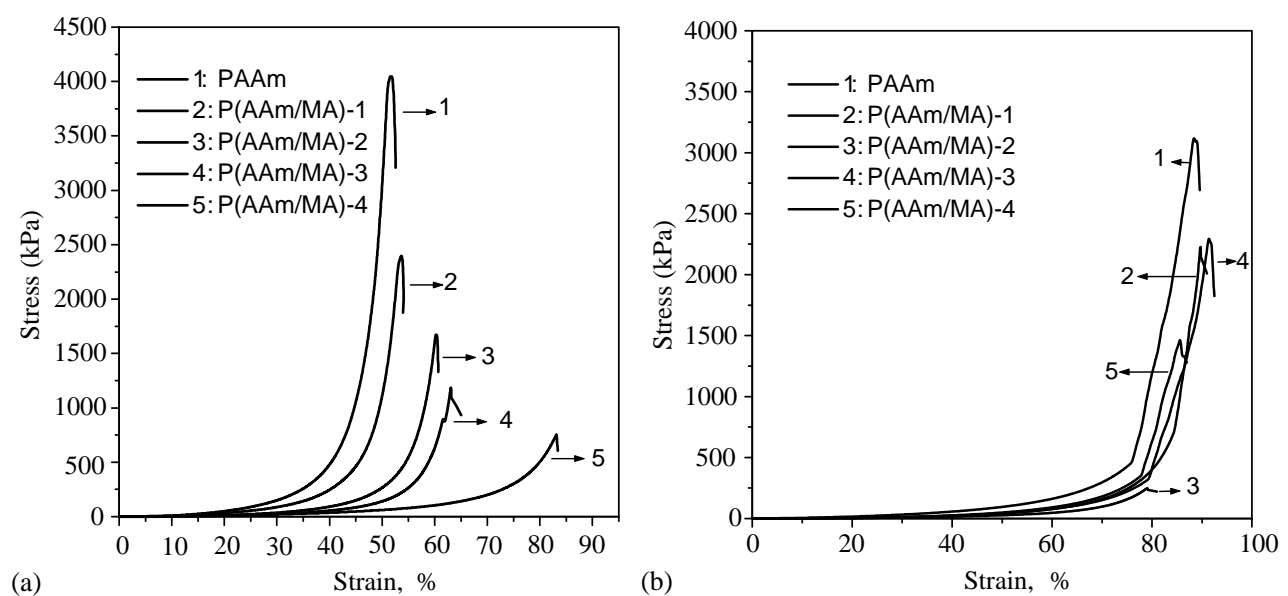

Fig. 3. Mechanical properties of $\mathrm{P}(\mathrm{AAm} / \mathrm{MA})$ hydrogels. Irradiation dose rate: (a) $3.0 \mathrm{kGy} / \mathrm{h}$, (b) $0.18 \mathrm{kGy} / \mathrm{h}$.

Table 2

Mechanical properties of $\mathrm{P}(\mathrm{AAm} / \mathrm{MA})$ hydrogels

\begin{tabular}{|c|c|c|c|c|c|c|}
\hline \multirow[b]{3}{*}{ Gel name } & \multicolumn{3}{|c|}{ Low dose rate $(0.18 \mathrm{kGy} / \mathrm{h})$} & \multicolumn{3}{|c|}{ Moderate dose rate $(3.0 \mathrm{kG} / \mathrm{h})$} \\
\hline & \multicolumn{3}{|c|}{ Maximum compressive value } & \multicolumn{3}{|c|}{ Maximum compressive value } \\
\hline & Strain $(\%)$ & Stress $(\mathrm{kPa})$ & Shear moduli $(\mathrm{kPa})$ & Strain $(\%)$ & Stress $(\mathrm{kPa})$ & Shear moduli $(\mathrm{kPa})$ \\
\hline PAAm & 88 & 3100 & 27.2 & 52 & 4047 & 126.0 \\
\hline $\mathrm{P}(\mathrm{AAm} / \mathrm{MA})-1$ & 89 & 2230 & 5.8 & 54 & 2396 & 71.1 \\
\hline $\mathrm{P}(\mathrm{AAm} / \mathrm{MA})-2$ & 79 & 250 & 7.5 & 60 & 1671 & 27.5 \\
\hline $\mathrm{P}(\mathrm{AAm} / \mathrm{MA})-3$ & 94 & 2290 & 8.2 & 63 & 1186 & 20.8 \\
\hline $\mathrm{P}(\mathrm{AAm} / \mathrm{MA})-4$ & 85 & 1460 & 9.2 & 83 & 755 & 17.4 \\
\hline
\end{tabular}

also an important parameter for controlling the network structure of a hydrogel system. Due to highly exothermic polymerization and cross-linking of $\mathrm{AAm} / \mathrm{MA} /$ water mixtures at $10 \mathrm{kGy} / \mathrm{h}$ dose rate homogeneous $\mathrm{P}(\mathrm{AAm} / \mathrm{MA})$ hydrogels with reproducible properties could not be prepared in this study. Not only high dose rate but also low dose rate irradiation endanger the formation of homogenous network formation of P(AAm/MA) hydrogels.

\section{References}

Akkaș, P., 1996. MS Thesis, Hacettepe University, Ankara, Turkey.
Güven, O., Sen, M., Karadaḡ, E., Suaraydin, D., 1999. A review on the radiation synthesis of copolymeric hydrogels for adsorption and separation purposes. Radiat. Phys. Chem. 56, 381-386.

Șen, M., Pekel, N., Güven, O., 1998. Radiation synthesis and characterization of n-vinyl-2-pyrrolidone/ $N$-allylthiourea hydrogels and their use in the adsorption of invertase. Die Angew. Macromol. Chem. 257, 1-6.

Șen, M., Uzun, C., Güven, O., 2000. Controlled release of terbinafine hydrochloride from $\mathrm{pH}$ sensitive poly (acrylamide/maleic acid) hydrogels. Int. J. Pharm. 203, 149-157.

Treloar, L.R.G., 1975. The Physics of Rubber Elasticity. Clarendon Press, Oxford. 